\title{
Competitive Opinion Maximization in Social Networks
}

\author{
Jianjun Luo \\ Worcester Polytechnic Institute \\ Department of Data Science \\ Worcester, USA \\ jluo@wpi.edu
}

\author{
Xinyue Liu \\ Worcester Polytechnic Institute \\ Department of Computer Science \\ Worcester, USA \\ xliu4@wpi.edu
}

\author{
Xiangnan Kong \\ Worcester Polytechnic Institute \\ Department of Computer Science \\ Worcester, USA \\ xkong@wpi.edu
}

\begin{abstract}
Influence maximization in social networks has been intensively studied in recent years, where the goal is to find a small set of seed nodes in a social network that maximizes the spread of influence according to a diffusion model. Recent research on influence maximization mainly focuses on incorporating either user opinions or competitive settings in the influence diffusion model. In many real-world applications, however, the influence diffusion process often involves both real-valued opinions from users and multiple parties that are competing with each other. In this paper, we study the problem of competitive opinion maximization, where the game of influence diffusion includes multiple competing products and the goal is to maximize the total opinions of activated users by each product. This problem is very challenging because it is \#P-hard and no longer keeps the property of submodularity. We propose a novel model, called ICOM (Iterative Competitive Opinion Maximization), that can effectively and efficiently maximize the total opinions in competitive games by taking user opinions as well as the competitor's strategy into account. Different from existing influence maximization methods, we inhibit the spread of negative opinions and search for the optimal response to opponents' choices of seed nodes. We apply iterative inference based on a greedy algorithm to reduce the computational complexity. Empirical studies on real-world datasets demonstrate that comparing with several baseline methods, our approach can effectively and efficiently improve the total opinions achieved by the promoted product in the competitive network.
\end{abstract}

Index Terms-influence maximization, social networks, competitive opinion maximization

\section{INTRODUCTION}

Motivated by many real-world applications (such as viral marketing), the problem of influence maximization has been studied intensively in recent years. The goal of influence maximization is to identify a small set of influential users in a social network, so that the coverage of an item (e.g., a product or a political view) is maximized in the influence diffusion. More formally, given a social network and a diffusion model, the influence maximization problem aims at selecting a small set of seed nodes that maximizes the spread of influence in

Permission to make digital or hard copies of all or part of this work for personal or classroom use is granted without fee provided that copies are not made or distributed for profit or commercial advantage and that copies bear this notice and the full citation on the first page. Copyrights for components of this work owned by others than ACM must be honored. Abstracting with credit is permitted. To copy otherwise, or republish, to post on servers or to redistribute to lists, requires prior specific permission and/or a fee. Request permissions from permissions@acm.org

ASONAM '19, August 27-30, 2019, Vancouver, Canada (C) 2019 Association for Computing Machinery.

ACM ISBN 978-1-4503-6868-1/19/08/\$15.00

https://doi.org/10.1145/3341161.3342899

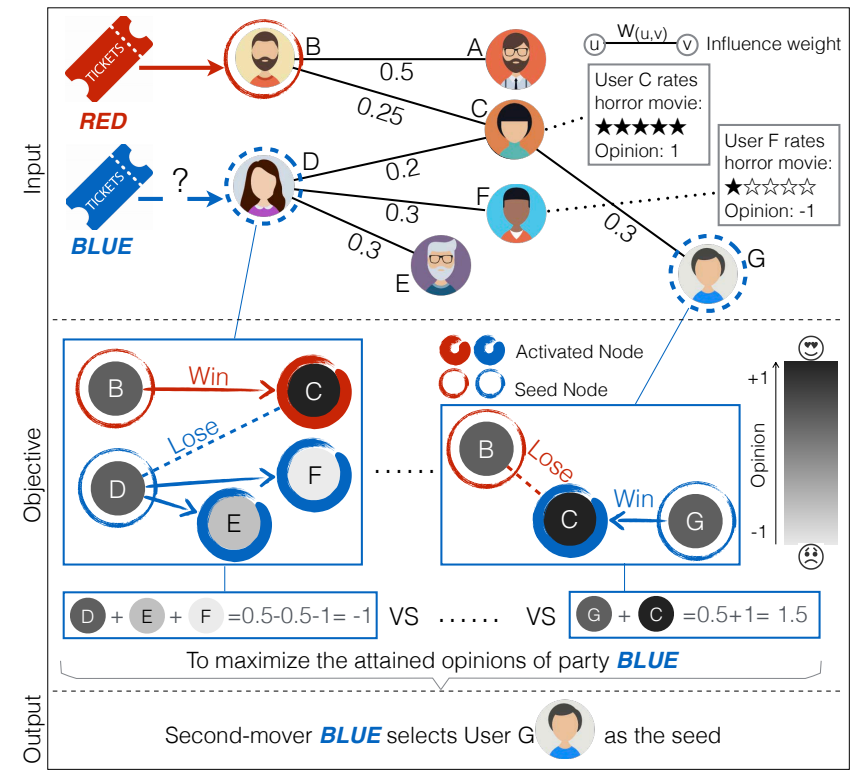

Fig. 1. An example of competitive opinion maximization. Given a social network and two parties (blue and red) promoting competing products (e.g., two horror movies), which users should the promoter (blue) pick as the seeds for viral marketing in two separate cases: (1) second-mover case: the seed users picked by the competitor (red) is known; (2) first-mover case: the promoter (blue) picks the seeds first, then the competitor (red) picks. Each user in the social network has a real-valued opinion (rating) towards the products. The two parties are competing to infect users in a competitive diffusion process. The goal of the promoter (blue) is to maximize the total ratings of its infected users. For simplicity, we ignore the first-mover case in this example.

the social network [19]. This problem is especially important in viral marketing. For example, with a limited budget for product promotion, a company wants to selectively choose a small set of users to distribute free samples, hoping that they will recommend the product to their friends, consequently increase the product sales or brand awareness by word-ofmouth marketing.

Conventional methods on influence maximization mainly focus on maximizing the user coverage in a social network, i.e., assuming that all users have identical utility scores. The more users get infected in the diffusion, the better the performance (e.g., higher sales or better reputation) is. Furthermore, most of the existing works focus on non-competitive settings, i.e., assuming that there is only one party in the diffusion process. However, in many real-world applications, neither of the assumptions above holds. The diffusion process often 


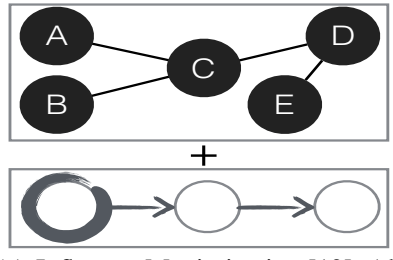

(a) Influence Maximization [19]: All users (nodes) have identical importance and the diffusion is noncompetitive.

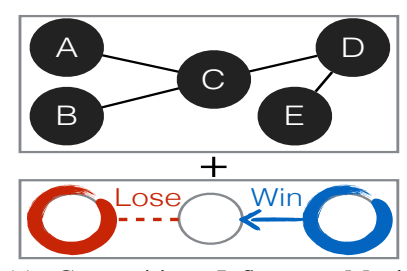

(c) Competitive Influence Maximization [2]: All users (nodes) have identical importance, but the diffusion is competitive.

Fig. 2. Comparison of four related problems.

involves multiple parties promoting competing items (e.g., products or political views) simultaneously and different users may have different opinions (or ratings) once infected in the diffusion. Each opinion (rating) is often a real-valued number, that can be negative. For example, in Fig. 1. two movie studios (blue and red) released two horror films at the same weekend. The two studios may want to compete for high ratings in movie reviews (e.g., IMDB ratings) in order to attract more viewers in later weeks. Each rating may be positive or negative, depending on whether the viewer loves or hates horror movies. An ideal viral-marketing strategy should focus on finding horror movie fans and those who can influence the most fans. Therefore, we define a new problem to solve these real-world problems.

In this paper, we study the competitive opinion maximization (COM) problem with a competitive linear threshold (CLT) model. In CLT model, each party propagates the same way as it does in the linear threshold model [19], but an activated node cannot be influenced again by another party, since people seldom adopt another homogeneous product in a short time. An inactive node which receives influence from different parties at the same time will be activated by the one who sends the highest influence weight. Formally, the competitive opinion maximization problem corresponds to selecting a small set of seed users as the optimal response to the observed or assumed opponent's choices of seeds. The objective of the selection is to maximize the total opinions gained after a competitive diffusion. Any two parties of the competition can be divided as a first mover and a second mover. A second mover simply makes its selection based on the known opponent's selection, but the first mover needs to search for optimal choices to maximize the total opinions under the disadvantages of being observed by its opponent. Since the influence spread computation is \#P-hard [10], the computation of opinions (spread with different utility scores) is at least \#P-hard. The problem of opinion maximization in CLT diffusion model has not been studied in this context so far.

The major research challenges on competitive opinion maximization are summarized as follows:

- User Opinion: One major challenge of the competitive opinion maximization problem is due to the real-valued opinions of the users. Conventional methods for influence maximization (as shown in Fig. 2(a) and Fig. 2(c) assume that all users in the social network have identical and positive utility scores. However, in competitive opinion maximization, user opinions can be negative and different users have different real-valued ratings. In such case, without considering opinions, a promotion strategy can lead to bad overall ratings. For example, horror films or cult films can be highly-rated by some viewers, but unpopular among mainstream audiences. It is important to target the appropriate customers because maximizing the spread of influence during the diffusion is no longer the optimal solution.

- Rational Competitors: Another challenge comes from the fact that multiple parties are competing in the market. If a user has already adopted an item, he or she will not accept another of the same type at the same time. We assume all the parties in the market are rational, which means they are likely to choose similar ideal customers if ignoring the competitors. The failure in a competition blocks the diffusion of influence and significantly lower the total opinions. A wise decision should take opponents' choices into consideration and estimate the outcome based on the possible failure. As shown in Fig. 2(c) and Fig. 2(d), a competitive diffusion model is necessary for the promoters to simulate the propagation of influence with competition.

- Scalability: Different from the problems that maximize the influence, our COM problem is neither submodular or monotonic, which means a standard greedy algorithm has no guarantee to the approximation ratio in [3]. Still, if the opponents' choices of seeds are observed, we can use a greedy algorithm as a heuristics to search through the network, since the standard greedy algorithm can achieve good empirical performance in non-submodular problems [12]. However, in some cases, the promoter can only make decisions based on the known opponent's strategy and will be observed by competitors as the first mover. Due to the passive position, a naive greedy algorithm for a first mover requires a lot of simulations, which is very slow for large networks. To address the problem, we utilize the inference from iterative simulation and design an efficient and effective heuristic algorithm.

In order to address the above challenges, we propose a novel solution, called ICOM (Iterative Competitive Opinion Maximization) method, to solve COM problem. By explicitly exploiting the users' opinions and the opponents' information, our ICOM method effectively finds a set of seeds to compete with other parties for total opinions with an iterative inference procedure. Different from conventional influence maximization methods, the proposed ICOM model exploits following information: (1) users' opinions; (2) observed competitor's 
TABLE I

IMPORTANT NOTATIONS.

\begin{tabular}{|c|c|}
\hline Symbol & Definition \\
\hline $\mathcal{V}=\left\{u_{1}, \cdots, u_{n}\right\}$ & the set of nodes \\
\hline $\mathcal{E}=\left\{e_{i} \in \mathcal{V} \times \mathcal{V}\right\}$ & the set of edges or links \\
\hline $\mathcal{W}=\left\{w_{i j} \mid i, j=1, \cdots, n\right\}$ & the set of link weights \\
\hline $\mathcal{P}=\left\{p_{1}, \cdots, p_{c}\right\}$ & of parties competing \\
\hline $\mathcal{S}=\left(\mathcal{S}^{1}, \mathcal{S}^{2}, \cdots, \mathcal{S}^{c}\right)$ & the set of seed user selections \\
\hline & $\begin{array}{l}\text { of } c \\
\text { user }\end{array}$ \\
\hline & $\begin{array}{l}\text { influence weight from } u_{j} \text { to } u_{i} \\
\text { type of competing item(product) } t\end{array}$ \\
\hline $\begin{array}{r}p_{v} \\
t \in[-1,1]\end{array}$ & $\begin{array}{l}\text { party(promoter) } v \\
u_{i} \text { 's opinion towards } i_{t}\end{array}$ \\
\hline$s_{i}^{v} \in[0,1]$ & the active status of $u_{i}$ \\
\hline $\mathcal{S}$ & the seed user selection of $p_{v}$ \\
\hline $\mathbf{o}_{t}=\left(o_{1 t}, o_{2 t}, \cdots, o_{n t}\right)$ & tor of opinions on \\
\hline $\mathbf{s}_{v}=\left(s_{1}^{v}, s_{2}^{v}, \cdots, s_{n}^{v}\right)$ & $\begin{array}{l}\text { the vector of status of users } \\
\text { activated by } p_{v}\end{array}$ \\
\hline
\end{tabular}

choice of seeds; (3) competitor's strategy. Empirical studies on real-world tasks demonstrate that the proposed iterative opinion maximization approach significantly boosts the performances in terms of total opinions achieved by the promoter with competitors in real-world datasets.

\section{PROBLEM Formulation}

\section{A. Concept Definitions}

The defined concepts will be used throughout this paper.

Definition 1 (Social Network): An online social network can be represented as $G(\mathcal{V}, \mathcal{E}, \mathcal{W})$, where nodes $\mathcal{V}=$ $\left\{u_{1}, \cdots, u_{n}\right\}$ is the set of users, $\mathcal{E}$ is the set of social links among users in $\mathcal{V}$ and $\mathcal{W}=\left\{w_{i j} \mid i=1, \cdots, n ; j=1, \cdots, n\right\}$ is the set of link weights. In the network, user(node) $u_{i}$ is influenced by its neighbor $u_{j}$ according to the weight $w_{i j}$.

Definition 2 (Opinions): $\mathbf{o}_{t}=\left(o_{1 t}, o_{2 t}, \cdots, o_{n t}\right)$ is the opinion vector of an item type $i_{t}$, where $o_{i t} \in[-1,1]$ represents the opinion of user $u_{i}$ towards the type of items that compete with item $t$.

Definition 3 (Party): There are multiple parties $\mathcal{P}=$ $\left\{p_{1}, \cdots, p_{c}\right\}$ promoting competing products in the network. These competing products are homogeneous and share common features, thus users will express the same opinions (preferences) on the competing items from different parties. In other words, these parties share the same opinion vector when they promote competing items.

Definition 4 (Active User Vector): Users who are influenced by Party $v$ to adopt the promoted item are defined to be activated by $p_{v}$, while others are inactive. Active user vector $\mathbf{s}_{v}=\left(s_{1}^{v}, s_{2}^{v}, \cdots, s_{n}^{v}\right)$ represents the activated status of all users in the network by party $p_{v}$. Given the target item type $i_{t}$, when user $u_{i}$ is activated by party $p_{v}$ and adopt the product with opinion $o_{i t}, s_{i}^{v}=1$, otherwise $s_{i}^{v}=0$ (inactivated).

Definition 5 (Seed Set): The decisions made by $c$ competing parties are represented as seed user set list $\mathcal{S}=\left(\mathcal{S}^{1}, \mathcal{S}^{2}, \cdots, \mathcal{S}^{c}\right)$, while $\mathcal{S}^{-v}=$ $\left(\mathcal{S}^{1}, \cdots, \mathcal{S}^{v-1}, \mathcal{S}^{v+1}, \cdots, \mathcal{S}^{c}\right)$ defines the known or predicted seed sets selected by all parties except $p_{v}$.

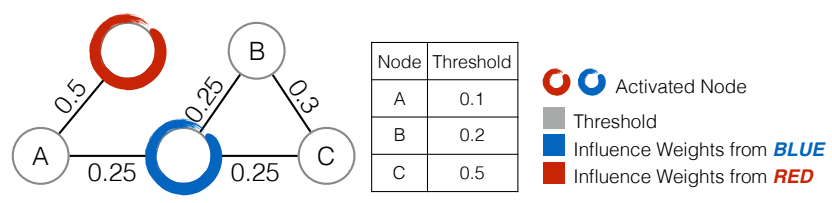

(a) $T=0$

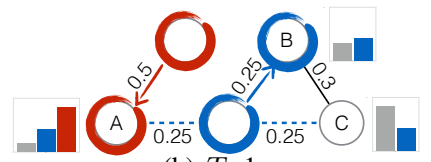

(b) $T=1$

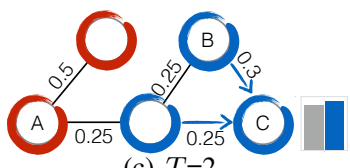

(c) $T=2$

Fig. 3. An example of CLT diffusion process. Two parties compete for the node A, the one with higher influence weight wins. Propagation stops when no new nodes can be activated.

\section{B. Problem Definition}

This paper focuses on the competitive LT model, but the proposed model shall be able to be adapted to competitive IC model.

Definition 6 (Competitive Linear Threshold): Competitive Linear Threshold (CLT) model is similar to the naive LT model, but with multiple participants. Each node $u_{i}$ in the network has an activation threshold $\theta_{i}$ chosen uniformly at random in $[0,1]$ and is possibly influenced by each neighbor $j$ with weight $w_{i j}$ such that $\sum_{j} w_{i j} \leq 1$. The influences of different parties start to propagate at the same time. Given a random choice of thresholds, at any timestamp $T$, if the total received weight of an inactive node $i$ from party $p_{v}$ 's activated neighbors is higher than that from other parties and $\theta_{i}$, then $u_{i}$ is activated by $p_{v}$ at timestamp $T+1$ (Fig. 3(b)). Once a node is activated by one party, it cannot be activated by any other party. When more than one party selects the same node as seed node or is eligible to activate the same node at the same timestamp $T$, e.g., $p_{1}$ and $p_{2}$, having $\sum_{j} w_{i j} s_{j}^{1}=\sum_{j} w_{i j} s_{j}^{2}>\theta_{i}$, node $i$ will be equally activated by both parties $p_{1}$ and $p_{2}$ at $T+1$, denoted as $s_{i}^{1}=s_{i}^{2}=\frac{1}{2}$. The influence process repeats until no new node becomes active by any party (Fig. 3(c)).

Definition 7 (Competitive Opinion Maximization): Given a network $G$, an item type $i_{t}$, corresponding opinion vector $\mathbf{o}_{t}$, the budget of seeds $k$ and the CLT diffusion model, the goal of the party $p_{v}$ in COM is to select a set of seed nodes $\mathcal{S}^{v}\left(\left|\mathcal{S}^{v}\right|=k\right)$ among $G$ to propagate the influence to maximize the expected total opinions towards its item achieved at the end of the diffusion:

$$
\mathcal{S}^{v}=\arg \max _{\mathcal{S}^{v}} \sigma\left(\mathcal{S}^{v}\right)
$$

We define

$$
\begin{aligned}
& \sigma\left(\mathcal{S}^{v}\right)=\sum_{\text {outcomes } X} \operatorname{Prob}(X) \cdot \sigma_{X}\left(\mathcal{S}^{v}\right) \\
& \sigma_{X}\left(\mathcal{S}^{v}\right)=s_{v}^{X} \cdot o_{t}
\end{aligned}
$$

where $\mathbf{s}_{v}^{X}$ is the active user vector $\mathbf{s}_{v}$ subject to a choice $X$ of node thresholds, denoting the set of all nodes reachable from any node in $\mathcal{S}^{v}$ for $p_{v}$, with the selections of other parties $\mathcal{S}^{-v}$. In other words, for $p_{v}$, the objective is to select seed set $\mathcal{S}^{v}$ to maximize the expected total opinions of eventually-influenced users $\sigma\left(\mathcal{S}^{v}\right)$. 


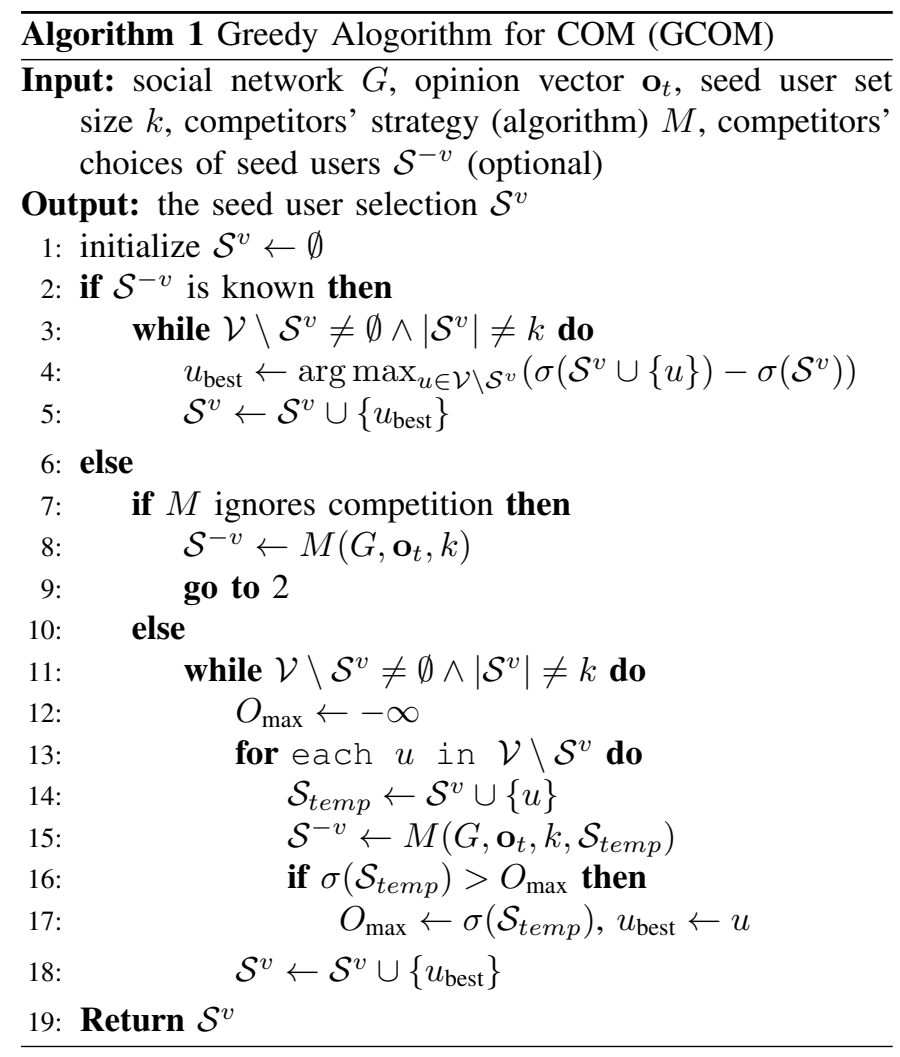

For simplicity, we set $|\mathcal{P}|=2$ in the following method description and experiment. We assume the opinions towards the items promoted by different parties are given and share the same opinion vector $\mathbf{o}_{t}$ for the specific item type $t$. This is consistent with the general case that users have relatively fixed preferences towards homogeneous products.

\section{The Proposed Method}

We first propose an adapted greedy algorithm (GCOM) by incorporating the competitive setting and opinion objective into the greedy IM algorithm. Then we propose an improved method (ICOM) to efficiently and effectively maximize the total opinions under CLT model.

It is worth noting that there are many improved algorithm to solve influence maximization problem [11], [17], [26]. Algorithms like CELF++ [14] and LDAG [8] are able to dramatically improve the efficiency under LT model. However, in this paper, we do not focus on improving the efficiency of seed selection algorithm in influence maximization as these studies. Instead, we propose a strategy that can solve COM problem effectively with opinions and opponent's information better than conventional IM strategies without additional consideration.

\section{A. Greedy Algorithm on COM}

Based on the accessible information, the parties can be classified into the first mover and the second mover. A second mover in the competition knows the competitor's exact choice of seed users $\mathcal{S}^{-v}$, while a first mover only knows the competitor's seed user selection strategy $M$.

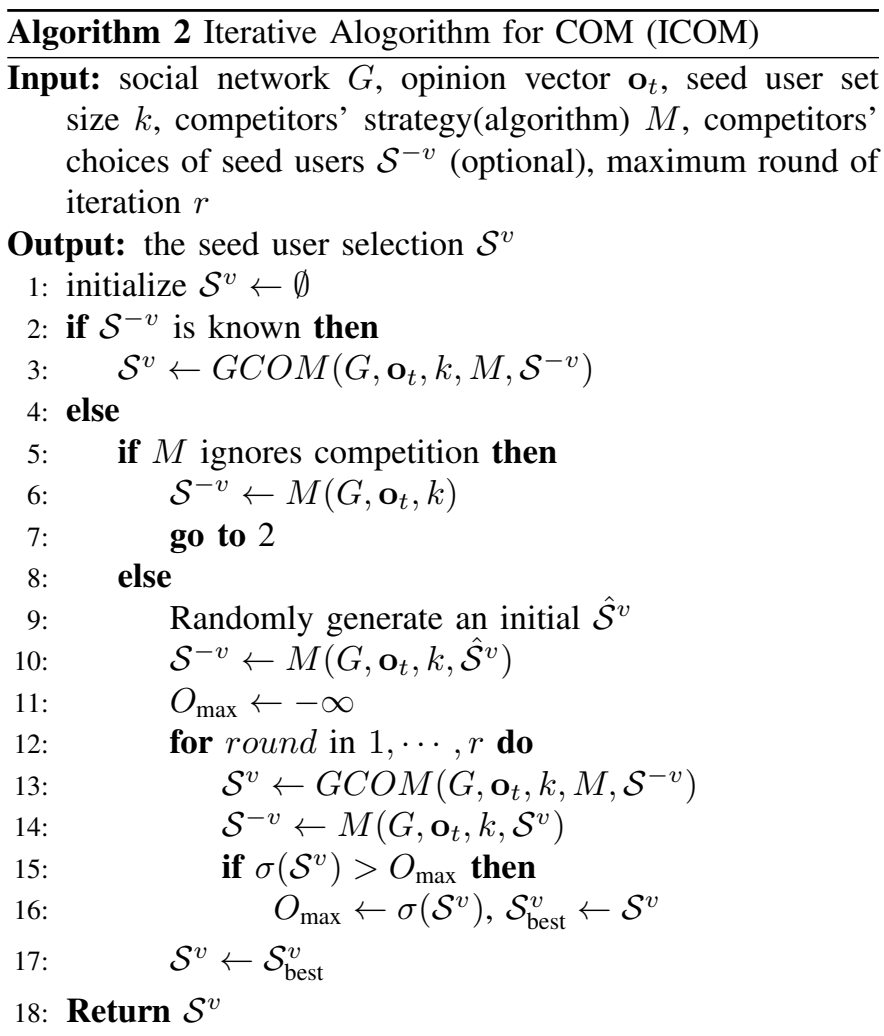

We use an analogous greedy algorithm GCOM to search the optimal response to the competitors' observed or simulated choices directly (Algorithm 11). If $p_{v}$ is a second mover who has observed the competitor's choices of seed users $\mathcal{S}^{-v}$, to form its own seed set, the promoter will search through all the nodes in $\mathcal{V}$ to greedily select the seeds. When searching for the $q$ th $(q \leq k)$ seed user, it tries every node that is not selected in the previous $q-1$ seeds to form different temporary set $\mathcal{S}^{v} \cup\{u\}$. Let the $q$ th seed of $p_{v}$ be the available node with the maximum simulated total opinions. The selecting process of a first mover who only knows the opponent's strategy $M$ is similar, but it needs to simulate the $\mathcal{S}^{-v}$ based on the temporary $\mathcal{S}^{v} \cup\{u\}$ repeatedly. To simplify the calculation, a first mover whose competitor uses fix strategy (ignores competition) can be seen as a second mover after one simulation. However, it will be impractical and not scalable for the first mover due to the extra cost of estimating the second mover's choice when the opponent's model considers competition. So we adapt GCOM by using iterative inference and propose an efficient and effective method to address the COM problem.

\section{B. The ICOM Method}

Now that we propose the ICOM method (Algorithm 2) to improve the GCOM algorithm by simplifying the work flow for the first mover. Reducing the simulation times required by a first mover whose opponent seed set is not fixed is a key point to tackle the limitation of the simple greedy method. Inspired by the tit for tat strategy in game theory, ICOM is a heuristic 


\section{IEEE/ACM International Conference on Advances in Social Networks Analysis and Mining}

TABLE II

SUMMARY OF EXPERIMENTAL DATASETS.

\begin{tabular}{lrrr}
\hline & \multicolumn{3}{c}{ Data Sets } \\
\cline { 2 - 4 } Characteristics & CiaoDVD & Flixster & Filmtrust \\
\hline \# Nodes & 2,740 & 5,320 & 1,642 \\
\# Links & $20.8 \mathrm{k}$ & $44.3 \mathrm{k}$ & $44.6 \mathrm{k}$ \\
\# Items & $13.1 \mathrm{k}$ & 3,470 & 2,071 \\
\# Ratings & $34.3 \mathrm{k}$ & $110 \mathrm{k}$ & $35.5 \mathrm{k}$ \\
Link type & directed & undirected & directed \\
Rating scale & {$[1,5]$} & {$[0.5,5]$} & {$[0.5,4]$} \\
\hline
\end{tabular}

method based on a small batch of multi-round simulation to approximate the strategic dominance.

Instead of considering every available node for the $q$ th seed to form the possible temporary seed set and then simulating the possible responses for the second mover, ICOM randomly chooses $k$ nodes as the very first seed set of the first mover $p_{v}$. Given the opponent's strategy $M$, we can easily infer the possible response $\mathcal{S}^{-v}$ from the competitor to the temporary $\mathcal{S}^{v}$. Then the iterative selection begins, and it includes the following steps: First, a new temporary $\mathcal{S}^{v}$ can be selected based on GCOM and the inferred $\mathcal{S}^{-v}$, i.e., first mover $p_{v}$ can search for an optimal response towards $\mathcal{S}^{-v}$ as a second mover. Then a new inferred $\mathcal{S}^{-v}$ can be predicted based on the new temporary $\mathcal{S}^{v}$ and the strategy $M$. The final active users of $p_{v}$ diffused by $\mathcal{S}^{v}$ after competing with $\mathcal{S}^{-v}$ will be used to estimate the total opinion $\sigma\left(\mathcal{S}^{v}\right)$ achieved in this round. Repeat such iterative inference until the maximum round $r$ is reached. We choose the $\mathcal{S}^{v}$ with the highest total opinion $O_{\max }$ in all these rounds as the seed set of $p_{v}$.

To simplify the problem, we assume competitor's selection model is known. If the model is not given, we can assume the competitor will take optimal strategy. According to the empirical results (section IV], this assumption generally can be seen as the case that both parties use the strategy combining opinions and competition.

\section{EXPERIMENTS}

\section{A. Data Collection}

We test our approach on three real-world networks with ratings (Summarized in Table II).

CiaoDVD: Ciao is a website for product reviews and price comparison. The dataset is crawled from the entire category of DVDs from the website [16]. The trust relationships between users are represented by the directed edges of the networks.

Flixster: Flixster is a website and a mobile app for movie information and ratings [1]. The users of Flixster link each other in the form of "friendship".

Filmtrust: FilmTrust is a small dataset crawled from the entire FilmTrust website in June, 2011. The website integrates "trust" social networks with movie ratings and reviews.

\section{B. Experiment Setting}

In CLT model, a user $u_{i}$ influences neighbors with certain weights. The weight of a directed link $e_{i j}$ measures the influence from $u_{j}$ to $u_{i}$. We calculate the weight of $e_{i j}$ using Jaccard coefficient, which is widely used in social influence analysis. The strength of relationship, i.e., the weight of link is defined as $\frac{\Gamma\left(u_{i}\right) \cap \Gamma\left(u_{j}\right)}{\Gamma\left(u_{i}\right) \cup \Gamma\left(u_{j}\right)}$. The threshold of users $\left[\theta_{1}, \ldots, \theta_{m}\right]$, is randomly generated from a uniform distribution within $[0,1]$. We can get complete ratings of an item from all the users by the incomplete rating vector in datasets. To predict unknown ratings based on observed ratings, we use the matrix factorization method for collaborative filtering following [20], [23], [25]. The rating of user $u_{i}$ towards item $t$ is approximated by the inner product of user profile $\mathbf{u}_{i}$ and item profile $\mathbf{v}_{j}$, which we can learn given the observed ratings. The approximated ratings which exceed the scope are replaced by the highest or lowest rating allowed in corresponding data set. We then convert the ratings to opinions using minmax normalization. The opinion $o_{i t}=2 \cdot \frac{r_{i t}-r_{\min }^{t}}{r_{\max }^{t}-r_{\min }^{t}}-1$, where $r_{\max }^{t}$ and $r_{\min }^{t}$ are the maximum and minimum rating of product $t$. This operation maps the range of ratings to the range -1 to 1 , which distinguishes the influence of positive or negative opinion towards the item, while original ratings in real-world dataset are always positive.

Given the network represented by the weighted adjacency matrix, and the opinions converted from ratings, we randomly choose three items from each dataset to perform the experiments. For each experiment, there are two parties in the market: a first mover and a second mover. There are five strategies for each party to take, i.e., for an item type in a network, there are 25 kinds of setting to simulate the possible results in a competitive market.

\section{Compared Methods}

In order to demonstrate the effectiveness of our ICOM approach, we test with following methods.

- Random: It is a baseline method that selects inactive nodes as seeds randomly.

- Influence Maximization (IM) [19]: It is a greedy method for influence maximization problem under linear threshold model. - Opinion Maximization (OM): It is an adaptive version of IM, which also greedily select seeds. Instead of selecting the node with largest marginal spread, it selects the one that can gain highest opinion.

- Competitive Influence Maximization (ICIM): It is a degenerative version of ICOM, which neglects the preferences of users on the target item, aiming to get wider spread. It takes the number of infected nodes as optimal objective instead of total opinions.

- Competitive Opinion Maximization (ICOM): It is the proposed method based on CLT diffusion model.

All methods except Random include a greedy method to select the next seed with the largest marginal objective, so IM, OM, ICIM are sensible to be the baseline of ICOM. We can improve the efficiency of these algorithms by adapting that common step in future study. The comparison shows the advantage of combining opinions and competitors information into the strategy for COM problem. All of the experiments are evaluated under competitive environment, even though Method Random, IM or OM selects seeds as if it is the only promoter in the diffusion. In different settings, we use corresponding strategies to get the seed nodes of the first mover and the second mover. We then use the CLT diffusion 
2019 IEEE/ACM International Conference on Advances in Social Networks Analysis and Mining

TABLE III

RESULTS OF DIFFERENT MODELS AS FIRST MOVERS. THE RESULTS ARE REPORTED AS "AVERAGE OPINIONS + (RANK)".

\begin{tabular}{|c|c|c|c|c|c|c|c|c|c|c|c|}
\hline \multirow[b]{2}{*}{ Competitor } & \multirow[b]{2}{*}{ Methods } & \multicolumn{3}{|c|}{ Ciao } & \multicolumn{3}{|c|}{ Flixster } & \multicolumn{3}{|c|}{ Filmtrust } & \multirow{2}{*}{$\begin{array}{l}\text { Avg. } \\
\text { Rank }\end{array}$} \\
\hline & & Item $C_{1}$ & Item $C_{2}$ & Item $C_{3}$ & Item $X_{1}$ & Item $X_{2}$ & Item $X_{3}$ & Item $T_{1}$ & Item $T_{2}$ & Item $T_{3}$ & \\
\hline Random+ & $\begin{array}{r}\text { Random } \\
\text { IM } \\
\text { OM } \\
\text { ICIM } \\
\text { ICOM }\end{array}$ & $\begin{array}{l}310.1(5) \\
583.4(3) \\
595.6(1) \\
564.0(4) \\
586.4(2)\end{array}$ & $\begin{array}{l}210.8(5) \\
372.0(3) \\
389.4(1) \\
353.7(4) \\
382.8(2)\end{array}$ & $\begin{array}{l}197.6(5) \\
355.6(2) \\
378.5(1) \\
341.0(4) \\
352.5(3)\end{array}$ & $\begin{array}{r}67.3(3) \\
28.0(5) \\
66.3(4) \\
100.1(2) \\
117.4(1)\end{array}$ & $\begin{array}{r}49.7(4) \\
18.7(5) \\
56.9(3) \\
91.2(2) \\
121.9(1)\end{array}$ & $\begin{array}{r}-90.0(4) \\
-51.4(3) \\
15.5(1) \\
-200.7(5) \\
-51.3(2)\end{array}$ & $\begin{array}{r}83.9(5) \\
130.5(4) \\
137.7(3) \\
166.9(2) \\
173.6(1)\end{array}$ & $\begin{array}{r}76.6(5) \\
120.7(4) \\
126.5(3) \\
152.9(2) \\
222.1(1)\end{array}$ & $\begin{array}{l}18.3(5) \\
28.3(4) \\
36.9(3) \\
37.9(2) \\
39.4(1)\end{array}$ & $\begin{array}{r}(4.6) \\
(3.7) \\
(2.2) \\
(3) \\
(1.6)\end{array}$ \\
\hline IM+ & $\begin{array}{r}\text { Random } \\
\text { IM } \\
\text { OM } \\
\text { ICIM } \\
\text { ICOM }\end{array}$ & $\begin{array}{r}76.8(5) \\
255.5(4) \\
287.2(3) \\
542.8(2) \\
553.2(1)\end{array}$ & $\begin{array}{r}51.5(5) \\
170.2(4) \\
299.0(3) \\
369.2(2) \\
378.5(1)\end{array}$ & $\begin{array}{r}48.3(5) \\
164.5(4) \\
248.8(3) \\
353.8(2) \\
364.2(1)\end{array}$ & $\begin{array}{r}163.9(4) \\
5.9(5) \\
185.6(3) \\
211.8(2) \\
226.6(1)\end{array}$ & $\begin{array}{r}132.0(4) \\
4.3(5) \\
157.7(3) \\
162.9(2) \\
179.7(1)\end{array}$ & $\begin{array}{r}-276.9(4) \\
-6.7(3) \\
15.7(2) \\
-348.8(5) \\
20.4(1)\end{array}$ & $\begin{array}{l}261.0(3) \\
171.1(5) \\
178.1(4) \\
398.1(2) \\
402.4(1)\end{array}$ & $\begin{array}{l}238.5(3) \\
157.1(5) \\
164.1(4) \\
364.4(2) \\
368.9(1)\end{array}$ & $\begin{array}{l}58.7(3) \\
37.2(5) \\
46.0(4) \\
89.6(2) \\
96.3(1)\end{array}$ & $\begin{array}{r}(4) \\
(4.4) \\
(3.2) \\
(2.3) \\
(1)\end{array}$ \\
\hline $\mathrm{OM}+$ & $\begin{array}{r}\text { Random } \\
\text { IM } \\
\text { OM } \\
\text { ICIM } \\
\text { ICOM }\end{array}$ & $\begin{array}{r}75.1(5) \\
347.2(3) \\
254.8(4) \\
565.2(2) \\
558.5(1) \\
\end{array}$ & $\begin{array}{r}48.0(5) \\
125.5(4) \\
174.8(3) \\
370.8(2) \\
380.2(1)\end{array}$ & $\begin{array}{r}46.0(5) \\
154.8(4) \\
169.0(3) \\
353.2(2) \\
358.2(1)\end{array}$ & $\begin{array}{r}140.1(3) \\
20.9(4) \\
3.1(5) \\
207.5(2) \\
226.1(1) \\
\end{array}$ & $\begin{array}{r}112.9(4) \\
10.0(5) \\
50.6(3) \\
160.7(2) \\
184.9(1) \\
\end{array}$ & $\begin{array}{r}-343.5(5) \\
-354.4(3) \\
3.3(2) \\
-354.4(3) \\
12.9(1)\end{array}$ & $\begin{array}{l}261.0(3) \\
171.0(5) \\
174.6(4) \\
395.0(2) \\
402.4(1)\end{array}$ & $\begin{array}{l}238.3(3) \\
158.1(5) \\
160.1(4) \\
362.7(2) \\
368.9(1)\end{array}$ & $\begin{array}{l}58.7(3) \\
37.4(5) \\
41.5(4) \\
88.4(2) \\
96.3(1)\end{array}$ & $\begin{array}{r}(4) \\
(4.2) \\
(3.6) \\
(2.1) \\
(1)\end{array}$ \\
\hline ICIM+ & $\begin{array}{r}\text { Random } \\
\text { IM } \\
\text { OM } \\
\text { ICIM } \\
\text { ICOM }\end{array}$ & $\begin{array}{r}11.6(5) \\
92.2(3) \\
78.2(4) \\
306.0(1) \\
300.8(2) \\
\end{array}$ & $\begin{array}{r}6.2(5) \\
37.2(4) \\
55.8(3) \\
210.8(2) \\
222.0(1)\end{array}$ & $\begin{array}{r}6.4(5) \\
38.2(4) \\
60.2(3) \\
196.0(1) \\
191.4(2)\end{array}$ & $\begin{array}{r}0.8(4) \\
-1.6(5) \\
5.6(3) \\
80.4(1) \\
73.7(2)\end{array}$ & $\begin{array}{r}1.3(4) \\
-1.6(5) \\
10.1(3) \\
59.1(2) \\
70.4(1)\end{array}$ & $\begin{array}{r}-1.4(4) \\
-0.8(3) \\
15.7(1) \\
-132.0(5) \\
14.2(2)\end{array}$ & $\begin{array}{r}75.3(3) \\
1.4(5) \\
8.6(4) \\
369.4(1) \\
231.3(2)\end{array}$ & $\begin{array}{r}68.8(3) \\
2.3(5) \\
8.3(4) \\
338.6(2) \\
338.9(1)\end{array}$ & $\begin{array}{r}15.8(3) \\
-0.3(5) \\
8.3(4) \\
78.6(2) \\
84.5(1)\end{array}$ & $\begin{array}{r}(4) \\
(4.3) \\
(3.2) \\
(1.9) \\
(1.6)\end{array}$ \\
\hline ICOM+ & $\begin{array}{r}\text { Random } \\
\text { IM } \\
\text { OM } \\
\text { ICIM } \\
\text { ICOM }\end{array}$ & $\begin{array}{r}9.8(5) \\
83.2(4) \\
94.5(3) \\
291.6(1) \\
287.6(2)\end{array}$ & $\begin{array}{r}3.6(5) \\
35.5(4) \\
40.8(3) \\
184.3(2) \\
195.5(1)\end{array}$ & $\begin{array}{r}4.7(5) \\
26.8(4) \\
53.2(3) \\
173.1(2) \\
184.4(1)\end{array}$ & $\begin{array}{r}-2.9(4) \\
-4.6(5) \\
4.2(3) \\
118.6(1) \\
105.2(2)\end{array}$ & $\begin{array}{r}-0.3(4) \\
-5.1(5) \\
3.9(3) \\
89.1(1) \\
81.4(2)\end{array}$ & $\begin{array}{r}-339.6(3) \\
-356.7(4) \\
14.7(1) \\
-358.1(5) \\
13.8(2)\end{array}$ & $\begin{array}{r}73.7(3) \\
1.4(5) \\
8.6(4) \\
362.8(1) \\
294.3(2)\end{array}$ & $\begin{array}{r}4.2(4) \\
2.1(5) \\
8.1(3) \\
74.2(2) \\
334.5(1)\end{array}$ & $\begin{array}{r}0.0(4) \\
-0.7(5) \\
7.9(2) \\
7.6(3) \\
67.5(1)\end{array}$ & $\begin{array}{r}(4.1) \\
(4.6) \\
(2.8) \\
(2) \\
(1.6)\end{array}$ \\
\hline
\end{tabular}

model to propagate the influence at the same time and output the total opinions of each party at the end as results. For models with randomness, their performances are measured by the 5-time average results.

\section{Performances on ICOM}

We first study the effectiveness of the proposed ICOM method on competitive opinion maximization. We report the total opinions achieved by the first mover in Table III] The performance is grouped by the opponent's strategy. Experiments are conducted over 9 randomly chosen items from 3 different networks. The budget of seed sets $k$ is 10 , while the inferring methods will only do 5 rounds inference $(r=5)$. Performance ranks of each model within the group are also listed. We use the average rank to compare the general performance of the model on item types with different popularity and in various networks. The performance of second mover are shown in Table IV grouped by the first mover's strategy.

The first observation we have in Table [II] and Table IV is as follows: almost all the methods that explicitly exploit the opinions of the item achieve higher total opinions than the baseline Random, and the corresponding degenerate method IM or CIM which only chases for the spread. These results support the importance of considering users' opinions and the opponent's seed selection jointly. Maximizing the number of activated users does not guarantee the total opinions gained for the items. In some cases, e.g., when diffusing a type of items with distinct feature that makes opinions from different people in stark contrast, or a kind of unpopular items that many people host negative opinions, the attempt to maximize the spread may even lead to a result worse than to select seed nodes randomly. We also observe that in many situations, the performances of models considering competition have a significant improvement compared with the ones that ignore. These results support our claim that in a multi-player

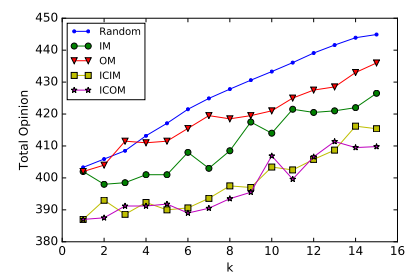

(a) Total opinions in the market

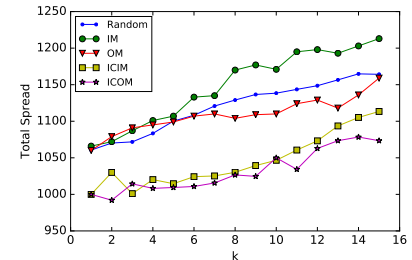

(b) Total spread in the market Fig. 4. Performances of two parties in Ciao when the second mover uses ICOM and the first mover chooses different strategies $(r=5)$.

propagation, taking the opponent's strategy into consideration usually enhances competitiveness. Making decision based on the known or simulated seeds of opponents avoids the possible failure in the competition or weaken performances brought by sharing the same customers with opponents. Thus, a market with multiple players should take competition into account and apply a more flexible and effective strategy to choose its seed users. These two main observations indicate the necessity and superiority of ICOM to achieve maximum opinions in a competitive environment. The overall results show that no matter as a first mover or a second mover and no matter what strategy the opponent takes, ICOM strategy, which exploits both opinion and competition information, is better than other baseline methods.

\section{E. Discussion of Parameters}

To have a better understanding of how the competitive strategy works in the market, we fix the second mover strategy and change the first mover strategy, to see how the overall market grows as the budget $k$ grows. Fig. 4 presents the performances from both participants in terms of opinions and influence spread. One can see that as the budget $k$ grows, the total gain over opinions or spread got by the two parties increases, which supports the intuition that increasing the budget improves the final performances. However, the choice 
2019 IEEE/ACM International Conference on Advances in Social Networks Analysis and Mining

TABLE IV

RESULTS OF DIFFERENT MODELS AS SECOND MOVERS.THE RESULTS ARE REPORTED AS "AVERAGE OPINIONS + (RANK)".

\begin{tabular}{|c|c|c|c|c|c|c|c|c|c|c|c|}
\hline \multirow[b]{2}{*}{ Competitor } & \multirow[b]{2}{*}{ Methods } & \multicolumn{3}{|c|}{ Ciao } & \multicolumn{3}{|c|}{ Flixster } & \multicolumn{3}{|c|}{ Filmtrust } & \multirow{2}{*}{$\begin{array}{l}\text { Avg } \\
\text { Rank }\end{array}$} \\
\hline & & Item $C_{1}$ & Item $C_{2}$ & Item $C_{3}$ & Item $X_{1}$ & Item $X_{2}$ & Item $X_{3}$ & Item $T_{1}$ & Item $T_{2}$ & Item $T_{3}$ & \\
\hline Random+ & $\begin{array}{r}\text { Random } \\
\text { IM } \\
\text { OM } \\
\text { ICIM } \\
\text { ICOM }\end{array}$ & $\begin{array}{l}273.9(5) \\
583.4(4) \\
595.6(3) \\
643.2(2) \\
655.8(1)\end{array}$ & $\begin{array}{l}185.5(5) \\
372.0(4) \\
389.4(3) \\
412.6(2) \\
429.4(1)\end{array}$ & $\begin{array}{l}181.6(5) \\
355.6(4) \\
378.4(3) \\
394.4(2) \\
416.3(1)\end{array}$ & $\begin{array}{r}121.4(4) \\
28.0(5) \\
66.2(3) \\
210.0(2) \\
229.2(1)\end{array}$ & $\begin{array}{r}98.9(4) \\
18.7(5) \\
56.9(3) \\
162.3(2) \\
182.5(1)\end{array}$ & $\begin{array}{r}-230.1(4) \\
-51.4(3) \\
15.5(2) \\
-345.1(5) \\
20.6(1)\end{array}$ & $\begin{array}{l}315.3(3) \\
130.5(5) \\
137.7 \text { (4) } \\
321.9 \text { (2) } \\
328.8(1)\end{array}$ & $\begin{array}{l}287.1 \text { (3) } \\
120.7 \text { (5) } \\
126.5 \text { (4) } \\
294.7 \text { (2) } \\
364.2(1)\end{array}$ & $\begin{array}{l}69.0(3) \\
28.3(5) \\
36.9(4) \\
72.3(2) \\
93.0(1)\end{array}$ & $\begin{array}{r}(4) \\
(4.4) \\
(3.2) \\
(2.3) \\
(1)\end{array}$ \\
\hline IM+ & $\begin{array}{r}\text { Random } \\
\text { IM } \\
\text { OM } \\
\text { ICIM } \\
\text { ICOM }\end{array}$ & $\begin{array}{r}76.8(5) \\
255.5(4) \\
287.2(3) \\
542.8(2) \\
553.2(1)\end{array}$ & $\begin{array}{r}51.5(5) \\
170.2 \text { (4) } \\
299.0 \text { (3) } \\
369.2(2) \\
378.5(1)\end{array}$ & $\begin{array}{r}48.3(5) \\
164.5(4) \\
248.8(3) \\
353.8(2) \\
364.2(1)\end{array}$ & $\begin{array}{r}163.9(4) \\
5.9(5) \\
185.6(3) \\
211.8(2) \\
226.6(1)\end{array}$ & $\begin{array}{r}132.0(4) \\
4.3(5) \\
157.7(3) \\
162.9(2) \\
179.7(1)\end{array}$ & $\begin{array}{r}-276.9(4) \\
-6.7(3) \\
15.7(2) \\
-348.8(5) \\
20.4(1)\end{array}$ & $\begin{array}{l}261.0(3) \\
171.1(5) \\
178.1(4) \\
398.1 \text { (2) } \\
402.4(1)\end{array}$ & $\begin{array}{l}238.5(3) \\
157.1 \text { (5) } \\
164.1 \text { (4) } \\
364.4(2) \\
368.9 \text { (1) }\end{array}$ & $\begin{array}{l}58.7(3) \\
37.2(5) \\
46.0(4) \\
89.6(2) \\
96.3(1)\end{array}$ & $\begin{array}{r}(4) \\
(4.4) \\
(3.2) \\
(2.3) \\
(1)\end{array}$ \\
\hline $\mathrm{OM}+$ & $\begin{array}{r}\text { Random } \\
\text { IM } \\
\text { OM } \\
\text { ICIM } \\
\text { ICOM }\end{array}$ & $\begin{array}{r}75.1(5) \\
347.2(3) \\
254.8(4) \\
565.2(2) \\
558.5(1)\end{array}$ & $\begin{array}{r}48.0(5) \\
125.5(4) \\
174.8(3) \\
370.8(2) \\
380.2(1)\end{array}$ & $\begin{array}{r}46.0(5) \\
154.8(4) \\
169.0(3) \\
353.2(2) \\
358.2(1)\end{array}$ & $\begin{array}{r}140.1(3) \\
20.9(4) \\
3.1(5) \\
207.5(2) \\
226.1(1) \\
\end{array}$ & $\begin{array}{r}112.9(4) \\
10.0(5) \\
50.6(3) \\
160.7(2) \\
184.9(1) \\
\end{array}$ & $\begin{array}{r}-343.5(5) \\
-354.4(3) \\
3.3(2) \\
-354.4(3) \\
12.9(1) \\
\end{array}$ & $\begin{array}{l}261.0(3) \\
171.0(5) \\
174.6(4) \\
395.0(2) \\
402.4(1)\end{array}$ & $\begin{array}{l}238.3(3) \\
158.1(5) \\
160.1 \text { (4) } \\
362.7(2) \\
368.9(1)\end{array}$ & $\begin{array}{l}58.7(3) \\
37.4(5) \\
41.5(4) \\
88.4(2) \\
96.3(1)\end{array}$ & $\begin{array}{r}(4) \\
(4.2) \\
(3.6) \\
(2.1) \\
(1)\end{array}$ \\
\hline ICIM+ & $\begin{array}{r}\text { Random } \\
\text { IM } \\
\text { OM } \\
\text { ICIM } \\
\text { ICOM }\end{array}$ & $\begin{array}{r}87.5(4) \\
92.2(3) \\
78.2(5) \\
299.1(2) \\
314.8(1) \\
\end{array}$ & $\begin{array}{r}64.1(3) \\
37.2(5) \\
55.8(4) \\
193.4(2) \\
215.6(1)\end{array}$ & $\begin{array}{r}58.2(4) \\
38.2(5) \\
60.2(3) \\
188.0(2) \\
210.2(1)\end{array}$ & $\begin{array}{r}84.8(2) \\
-1.6(5) \\
5.6(4) \\
101.0(1) \\
76.5(3)\end{array}$ & $\begin{array}{l}55.7(3) \\
-1.6(5) \\
10.1(4) \\
83.8(1) \\
63.2(2)\end{array}$ & $\begin{array}{r}-124.7(4) \\
-0.8(3) \\
15.7(2) \\
-172.8(5) \\
20.6(1)\end{array}$ & $\begin{array}{r}230.1(1) \\
1.4(5) \\
8.6(4) \\
24.6(3) \\
31.8(2)\end{array}$ & $\begin{array}{r}210.0(2) \\
2.3(5) \\
8.3(4) \\
21.4(3) \\
286.7(1)\end{array}$ & $\begin{array}{r}49.7(2) \\
-0.3(5) \\
8.3(4) \\
8.5(3) \\
81.9(1)\end{array}$ & $\begin{array}{l}(2.8) \\
(4.6) \\
(3.8) \\
(2.4) \\
(1.4)\end{array}$ \\
\hline ICOM+ & $\begin{array}{r}\text { Random } \\
\text { IM } \\
\text { OM } \\
\text { ICIM } \\
\text { ICOM }\end{array}$ & $\begin{array}{r}73.6(5) \\
83.2(4) \\
94.5(3) \\
302.4(2) \\
310.3(1)\end{array}$ & $\begin{array}{r}49.2(3) \\
35.5(5) \\
40.8(4) \\
178.4(2) \\
208.4(1)\end{array}$ & $\begin{array}{r}62.4(3) \\
26.8(5) \\
53.2(4) \\
192.6(2) \\
198.3(1)\end{array}$ & $\begin{array}{r}90.7(2) \\
-4.6(5) \\
4.2(4) \\
105.0(1) \\
83.6(3)\end{array}$ & $\begin{array}{r}42.3(3) \\
-5.1(5) \\
3.9(4) \\
75.4(1) \\
70.9(2)\end{array}$ & $\begin{array}{r}-264.7(3) \\
-356.7(5) \\
14.7(1) \\
-353.6(4) \\
14.3(2)\end{array}$ & $\begin{array}{r}228.5(1) \\
1.4(5) \\
8.6(4) \\
162.3(2) \\
100.7(3)\end{array}$ & $\begin{array}{r}145.4(1) \\
2.1(5) \\
8.1(4) \\
22.7(3) \\
26.7(2)\end{array}$ & $\begin{array}{r}53.2(1) \\
-0.7(5) \\
7.9(3) \\
4.4(4) \\
19.4(2)\end{array}$ & $\begin{array}{l}(2.4) \\
(4.9) \\
(3.4) \\
(2.3) \\
(1.9)\end{array}$ \\
\hline
\end{tabular}

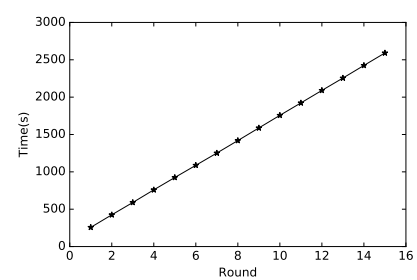

(a) Time

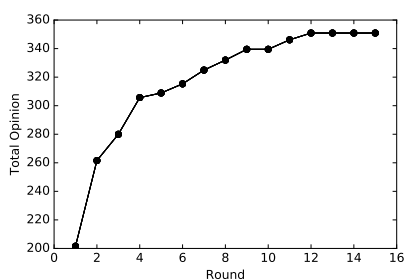

(b) CiaoDVD

Fig. 5. Influence of parameter round $r$

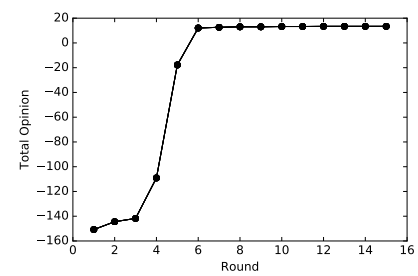

(c) Flixster

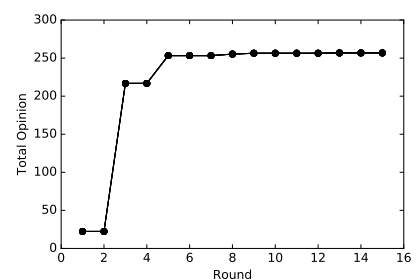

(d) Filmtrust of the first mover model makes the outcome different, while the second mover's fixed strategy is ICOM. As discussed in the previous section, the first mover will achieve more total opinions if it takes a competitive strategy. But from the figure, a competitive strategy will significantly decreases the second mover's gain and the overall gain of both parties. Therefore when both parties try to take a competitive strategy, maximizing its own gain, the overall market in terms of opinions or spread would decline.

Then we present the influence of different maximum number of round $r$ on the performance of ICOM model. Fig. 5 shows the convergence of the proposed ICOM by testing the performance after each iteration step. We randomly select an item from each of the datasets to run an experiment that both players are using ICOM, and each setting repeats 5 times to measure the performance. The budget $k$ is 10 and the maximum tested round $r$ is 15. Fig. 5(a) demonstrates the relationship between $r$ and the cost of time in the experiment of Ciao dataset, while other two figures are similar and omitted. It indicates that the time cost of ICOM linearly grows along with the parameter $r$. Fig. 5(b) to 5(d) show the total opinions achieved along with different $r$. The performances of the ICOM converge very fast after a few iterations. From these results, we see that the performance of ICOM is not sensitive to the maximum number of rounds as long as $r$ is assigned with a modest number. Although a global optimal result is not guaranteed, it has a relatively good performance and is suitable for balancing the performance and the cost. Thus in our previous experiment, we use 5 as the default maximum number of rounds. This also supports our intuition that using inference makes the cost of time controllable and exploiting competitor's selection is important and effective for competitive opinion maximization.

\section{RELATED WORK}

To the best of our knowledge, this paper is the first work on competitive opinion maximization. Our work is related to influence maximization, opinion maximization and competitive influence maximization.

Influence Maximization: Influence maximization (IM) is to study how to choose a small set of seed nodes in a network, which has the best opportunity to influence the most number of nodes through a given diffusion model. In [19] Kempe et al. obtained the first provable approximation guarantee for the two basic stochastic influence cascade models they proposed, the independent cascade (IC) model and the linear threshold (LT) model. Chen et al. [8]-[10] designed a scalable algorithm for the IC model that can handle large-scale social networks and proposed the first scalable IM algorithm tailored for the LT model. Instead of using heuristics to estimate the spread like [15], an efficient algorithm called CELF proposed by Leskovec et al. [21] exploits submodularity and dramatically improves the efficiency of the greedy algorithm for IM problem. 


\section{IEEE/ACM International Conference on Advances in Social Networks Analysis and Mining}

Opinion Maximization: Instead of selecting the most influential nodes, the aim of opinion maximization is to make the item favorable and get more positive opinions. Chen $e t$ al. [7] proposed a model that incorporates the emergence and propagation of negative opinions into the IC model to maximize the expected number of positive activated nodes. Zhang et al. [27] considered the negative and neutral opinions, proposed an adapted IC model to maximize the total opinions of activated users. Gionis et al. [13] assumed the opinions of individuals get formed dynamically by the mutual influence of internal opinions and the neighbors' opinions. Liu et al. [24] studied a multi-round single-party opinion maximization problem, where they attempts to find the optimal set of seeds in each round of promotion to maximize the total opinion spread in the network based on the opinion observed.

Competitive Influence Maximization: The problem is to study the simultaneous propagation of multiple items in a social network. The solutions for competitive influence maximization can basically be classified into two types: opponent strategy known or opponent strategy unknown. For the first type, a popular solution is to minimize the opponent's influence, i.e., Influence Blocking Maximization [3], [4], [18]. Carnes et al. [5] studied the competitive influence maximization problem from a follower's perspective, i.e., finding a best response to the first mover's selection. For the second type, Bharathi et al. [2] proposed a natural generalization of the IC model and used game theory to study the diffusion with multiple competing items. Chen et al. [6] proposed a data-driven approach to study the multi-player influence maximization and proposed a game to collect the picking strategies from human or AI to analysis. Lin et al. [22] proposed a learning-based framework using reinforcement learning and game theory to address the multi-round competitive influence maximization problem. Zhang et al. studied the maximization problem of multiple competing or complementary products in a social network at the same time in [28].

\section{CONCLUSION}

In this paper, we study the competitive opinion maximization (COM) problem and propose a novel method ICOM to address it. Our method is based on the CLT diffusion model, where different parties compete to activate nodes. ICOM estimates the users' opinions towards the target type of items (competing items) and optimizes the seed selection collectively by exploiting the information from competitors. ICOM also utilizes the iterative inferences to improve the performance of opinions and reduce the time complexity when competitors' seed selections are unknown. Based on three real-world social networks, the experiment results validate the effectiveness and efficiency of our proposed model ICOM.

\section{ACKNOWLEDGMENT}

This work is supported in part by National Science Foundation through grant IIS-1718310.

\section{REFERENCES}

[1] S. Bhagat, A. Goyal, and L. V. Lakshmanan. Maximizing product adoption in social networks. In WSDM '12, pages 603-612, 2012.

[2] S. Bharathi, D. Kempe, and M. Salek. Competitive influence maximization in social networks. Internet and Network Economics, pages 306-311, 2007.

[3] A. Borodin, Y. Filmus, and J. Oren. Threshold models for competitive influence in social networks. In WINE '10, pages 539-550, 2010.

[4] C. Budak, D. Agrawal, and A. El Abbadi. Limiting the spread of misinformation in social networks. In $W W W^{\prime}, 11$, pages 665-674, 2011.

[5] T. Carnes, C. Nagarajan, S. M. Wild, and A. Van Zuylen. Maximizing influence in a competitive social network: a follower's perspective. In ICEC '07, pages 351-360, 2007.

[6] H.-H. Chen, Y.-B. Ciou, and S.-D. Lin. Information propagation game: a tool to acquire humanplaying data for multiplayer influence maximization on social networks. In KDD '12, pages 1524-1527, 2012.

[7] W. Chen, A. Collins, R. Cummings, T. Ke, Z. Liu, D. Rincon, X. Sun, Y. Wang, W. Wei, and Y. Yuan. Influence maximization in social networks when negative opinions may emerge and propagate. In SDM '11, pages 379-390, 2011.

[8] W. Chen, C. Wang, and Y. Wang. Scalable influence maximization for prevalent viral marketing in large-scale social networks. In $K D D$ ' 10 , pages 1029-1038, 2010.

[9] W. Chen, Y. Wang, and S. Yang. Efficient influence maximization in social networks. In $K D D$ '09, pages 199-208, 2009.

[10] W. Chen, Y. Yuan, and L. Zhang. Scalable influence maximization in social networks under the linear threshold model. In ICDM '10, pages 88-97, 2010.

[11] C.-Y. Chiang, L.-H. Huang, B.-J. Li, J. Wu, and H.-G. Yeh. Some results on the target set selection problem. Journal of Combinatorial Optimization, 25(4):702-715, 2013.

[12] A. Das and D. Kempe. Submodular meets spectral: greedy algorithms for subset selection, sparse approximation and dictionary selection. In ICML '11, pages 1057-1064. Omnipress, 2011.

[13] A. Gionis, E. Terzi, and P. Tsaparas. Opinion maximization in social networks. In SDM '13, pages 387-395, 2013.

[14] A. Goyal, W. Lu, and L. V. Lakshmanan. Celf++: optimizing the greedy algorithm for influence maximization in social networks. In $W W W$ ' 11 Companion, pages 47-48. ACM, 2011.

[15] A. Goyal, W. Lu, and L. V. Lakshmanan. Simpath: An efficient algorithm for influence maximization under the linear threshold model. In ICDM '11, pages 211-220. IEEE, 2011.

[16] G. Guo, J. Zhang, D. Thalmann, and N. Yorke-Smith. Etaf: An extended trust antecedents framework for trust prediction. In ASONAM '14, pages $540-547,2014$.

[17] X. He and D. Kempe. Robust influence maximization. In $K D D$ ' 16 , 2016.

[18] X. He, G. Song, W. Chen, and Q. Jiang. Influence blocking maximization in social networks under the competitive linear threshold model. In SDM '12, pages 463-474, 2012.

[19] D. Kempe, J. Kleinberg, and É. Tardos. Maximizing the spread of influence through a social network. In $K D D$ '03, pages 137-146, 2003

[20] Y. Koren, R. Bell, and C. Volinsky. Matrix factorization techniques for recommender systems. Computer, 42(8), 2009.

[21] J. Leskovec, A. Krause, C. Guestrin, C. Faloutsos, J. VanBriesen, and N. Glance. Cost-effective outbreak detection in networks. In $K D D$ ' 07 , pages 420-429. ACM, 2007.

[22] S.-C. Lin, S.-D. Lin, and M.-S. Chen. A learning-based framework to handle multi-round multi-party influence maximization on social networks. In $K D D$ '15, pages 695-704, 2015.

[23] X. Liu, C. Aggarwal, Y.-F. Li, X. Kong, X. Sun, and S. Sathe. Kernelized matrix factorization for collaborative filtering. In SDM '16, pages 378386. SIAM, 2016

[24] X. Liu, X. Kong, and P. Yu. Active opinion maximization in social networks. In $K D D$ ' $18,2018$.

[25] X. Liu, Y. Song, C. Aggarwal, Y. Zhang, and X. Kong. Bicycle: Item recommedation with life cycles. In ICDM '17, pages 297-306, 2017.

[26] F. Morone and H. A. Makse. Influence maximization in complex networks through optimal percolation. Nature, 524(7563):65, 2015.

[27] H. Zhang, T. N. Dinh, and M. T. Thai. Maximizing the spread of positive influence in online social networks. In ICDCS '13, pages 317-326, 2013.

[28] J. Zhang, S. Wang, Q. Zhan, and S. Y. Philip. Intertwined viral marketing in social networks. In ASONAM '16, pages 239-246, 2016. 